in children differ from those in adults (Snider WD et al. Ann Neurol 1983; 14: 403). The incidence of CNS involvement is higher in children but unlike adult patients, CNS opportunistic infections appear to be uncommon, occurring in only $8 \%$ of the children in this study. A progressive dementing encephalitis (AIDS dementia complex) accompanied by regional cerebral metabolic alterations is the chief neurological complication of AIDS in adult cases (Rottenberg DA et al. Ann Neurol 1987; 22: 700). Peripheral neuropathy with painful dysesthesias and retinopathy with cotton-wool spots reported in $16 \%$ and $20 \%$, respectively, of adult cases were not recognized in the pediatric group. The static encephalopathy diagnosed in 17 (28\%) children was cause for optimism but this was tempered by a later progressive course in 5 similar cases. The incidence of potential AIDS cases in children may be higher than expected: a recent survey showed that 1 in 61 babies born in $\mathrm{NY}$ City carried antibodies to AIDS virus, indicating that their mothers were infected. About $40 \%$ of infants showing antibodies are estimated to be infected and may develop AIDS.

\title{
ACUTE VIRAL ENCEPHALITIS
}

In a retrospective study of children with viral encephalitis admitted to the University Paediatric Unit, queen Mary Hospital, Hong Kong, during the past 10 years, 57 satisfied the diagnostic criteria. The presumed viral etiology was determined in 15 (26\%) patients of whom 9 had post-infectious encephalitis (mumps-4, measles-1, rubella-1, influenza A-2, influenza B-1). The viruses isolated were: influenza ( 3 cases), Coxsackie (2), adenovirus (2), mixed adenovirus and cytomegalovirus (1), and herpes simplex (1). Presenting neurologic features included focal signs (33\%), convulsion (30\%), headache (25\%), drowsiness (18\%), nuchal rigidity (10\%), and coma (9\%). Seven (12\%) who developed status epilepticus within $24 \mathrm{hrs}$ of admission died subsequently. A total of 16 (28\%) died, and 5 were less than 1 year old. Indicators of a poor outcome were an onset in infancy and rapid deterioration in the level of consciousness.

Recovery was complete in $76 \%$ of 41 survivors; focal neurological deficits remained in 298 and epilepsy in $4 \%$ of 31 with sequelae. Eight children with suspected herpes simplex encephalitis on admission were treated with Acyclovir and none died; one in whom the diagnosis was confirmed developed spastic quadriplegia, mental retardation, and infantile spasms. (Wong V, Yeung CY. Acute viral encephalitis in children. Aust Paediatr J 1987; 23: 339-342).

COMEENr. Unlike previous reports from the USA and Scandinavia which have emphasized herpes simplx virus (HSE) as a major cause of acute sporadic viral encephalitis, only 1 case was identified in this study and none of the 16 patients who died had histological evidence of herpes simplex infection. Brain biopsy, a controversial diagnostic test, was not performed. A consensus panel of the Jrnl of Pediatric Infectious Disease has recommended 
early brain biopsy as a prerequisite for diagnosis and treatment of HSE. This view is supported by Hanley et al, Johns Hopkins Hospital, but is considered invalid by Fishman RA, Univ of California, San Francisco (Arch Neurol 1987; 44: 1289-1292). Brett EM, at the Hospital for Sick Children, Great Ormond Street, London, has spoken against routine diagnostic brain biopsy for suspected HSE in children ( $\mathrm{Br}$ Med J 1986; 293: 1388). Noninvasive magnetic resonance imaging (MRI) may provide early diagnosis without the immediate risks of biopsy and its later complications (Schroth G et al. Neurology 1987; 37: 179).

\section{NEUROCUTANEOUS SYNDROMES}

\section{TUBEROUS SCLEROSIS}

A 14-year-old girl with bilateral renal cell carcinoma (hypernephroma) complicating a previously unrecognized tuberous sclerosis is reported from Cornell Univ Med College, NY, and North Shore Univ Hosp, Manhasset, NY, together with a review of 6 similar cases culled from the literature. The patient presented with a 6-month history of progressive weight loss and anemia. She had an acne-like rash on her face and a nodule on her tongue. Her father and paternal uncle were institutionalized for convulsive and psychiatric disorders. Abdominal sonogram and CAT scan showed a large mass arising from the right kidney and a smaller mass in the left kidney. Areas of sclerosis and periventricular calcifications were found in skull X-rays and CAT scan of the head. Renal cell carcinoma and hamartomatous nodules were diagnosed at surgery. Epithelial-lined cysts of the kidney and adenoma sebaceum of the face were typical of tuberous sclerosis. All abnormal hematological and chemistry values, including hypercalcemia, anemia, thrombocytopenia, and hypoalbuminemia returned to normal and the patient was asymptomatic at 3 years after surgery. (Weinblatt ME et al. Renal cell carcinoma in patients with tuberous sclerosis. Pediatrics 1987; 80: 898-903).

COMIENT. Tumors occur more commonly in the kidney than in any other organ as a manifestation of tuberous sclerosis, their frequency being estimated at $80 \%$ (Critchley M, Earl CJC. Brain 1932; 55: 311). Hamartoma or multiple angiomyolipoma is the most frequent variety but hypernephroma, liposarcoma, adenosarcoma, myosarcoma, and perithelioma are also described (Wilson SAK. Neurology 1955, Williams and Wilkins, Baltimore). Mostly bilateral and often multiple, they sometimes undergo cystic degeneration. Of 29 cases of tuberous sclerosis reported by Fowler JS and Dickson WEC (Quart J Med 1910; 4: 43), 17 (58\%) had renal tumors.

The present authors recommend that all patients with tuberous sclerosis should have periodic sonography of the kidney and frequent urinalysis, especially in adolescence and adult life when the incidence of renal masses begins to increase. Fortunately, 